\title{
Athletic Meeting Grouping Lane Dividing Algorithm and System Design
}

\author{
Jian Pan \\ School of Education and Sports, Bohai University, Jinzhou, 121013, China
}

\begin{abstract}
With the development of computer and network technology, athletic meeting management is constantly toward modernization, and in the popular athletic meeting management software, the grouping lane dividing of track events is fixed eight lanes, scheduling algorithm is fixed, and there are some flaws. Therefore, researching grouping lane dividing algorithm and system design has important significance. Firstly, studies the athletic meeting grouping lane dividing algorithm and the basic idea, includes specific algorithmic process; secondly studies the system design on grouping lane dividing, including system flow chart; finally studies the logical design in the database design of grouping lane dividing system, this paper mainly embodied in the form of table structure. The contents of this paper improve the efficiency of the athletic meeting management and make up its shortcomings.
\end{abstract}

Keywords-Athletic meeting; grouping lane dividing; scheduling algorithm; system design; logical design.

\section{INTRODUCTION}

With the progress of the era and the rapid development of social productive forces, new technologies are emerging, the information is rapidly expanding, the entire human society has become information-based society, people take advantage of and process the information and data has entered automation, networking and socialization stage. Track and field as a basis for all sports, whether it is a high level of competition (such as the Olympics, Asian Games, Games, etc.), or is the grass-roots and universities, primary and secondary school track meet. Participation of the masses with high enthusiasm, it is to improve the health condition and to carry out national fitness campaign [1-3]. Athletic meeting choreography work is a very tedious work, traditional manual scheduling operation has low efficiency and low accuracy, copying task is heavy, heavy workload, requires a lot of manpower and resources. The choreography of order book computer-implemented makes organizational manual tedious choreography work can be achieved by a simple operation, so that the athletic meeting organization and management becomes simple, efficient, and flexible.

At present, in the various sports management software, for the question that grouping lane dividing in the track and field project it is fixed eight mostly, based the order of athlete registration to set up pass, this arrangement is not very scientific method, but also unreasonable, sometimes resulting in the final group only one or two players, while other groups were eight athletes; or creating more than athletes ranked in the same category competition, some athletes always on a fixed pass to match in front or in end [4]. Organization of the athletic meeting always uses manual registration, grouping lane dividing, ranking and scoring model, due to this model has a heavy workload and error prone. Therefore, this paper proposes a grouping lane dividing algorithm, it can effectively solve the questions existed in grouping lane dividing in the sports track project.

\section{GROUPING LANE DIVIDING ALGORITHM}

In the grouping lane dividing problems, grouping is based on competition rules, athletes participating in the project are grouped and generates a grouping order book, require the athlete in same unit not in the same group, the number of athletes in each group in the same project basically tend to balance, lane dividing is to add the athletes to a different passes, at the same time, the athletes in same unit cannot be on the same pass. In the athletic meeting project category, "Track 100" project requires grouping lane dividing, "Track $800 "$ project only grouping, regardless of lane dividing, "Tin Cup" project is same. "Track 100" project grouping lane dividing requires participating pass in each group uniform; the number of grouping in "track 800" is set in accordance with the requirements of the referee committee, the number of grouping in "Tin Cup" is set up according to ground, the participants of each group are randomly generated [5].

Randomized grouping algorithms: Firstly, statistics the number of participants (number of participants expressed by $m$ ), and determine the maximum number of each group in each item (namely, channel number $n$ );

Secondly, according to the number of participants and the number of people in each group determine the number of grouping (grouping indicated by $g$ ). Discuss two cases, when the number of participants is divisible by the number of people in each group, then the grouping number is the that the number of participants divided by the number of people in each group; if the number of participants cannot be divisible by the number in each group, then the grouping number is the that the number of participants divided by the number of people in each group and plus 1, its formula as follows:

$$
g=\left\{\begin{array}{c}
m / n \\
{[m / n]+1}
\end{array}\right\}
$$

Again, to index or sort by unit for the athletes; 
Finally, using number algorithm gives the affiliated group number for each athlete.

Lane dividing algorithm: first step, to determine the initial channel number $a$, the initial channel number is determined by the number of participants and the number of grouping, the formula is as follows,

$$
a=[\mathrm{m} / \mathrm{g}]
$$

The second step, to determine the highest passes $d$, the maximum passes is based on the initial pass $a$ plus 1 , namely $d=a+1$;

The third step is to determine the remaining number $s$, the remaining number is the differential value between the total number of participants and the actual number of participants in each group, the actual number of participants in each group is the product that the number of groups and the number of the initial channel number, namely $s=m-g \times a$;

It is noteworthy that, because the participating units of participants in each group cannot be repeated, and participants in each group were randomly generated for each channel, so the formula of random recording number $x$ for the athletes as follows:

$$
x=\operatorname{int}(\operatorname{rand}() \times(b-1))+1
$$

In which, $b$ is the current total number in projects the table (the total number of records), because plus one in group lane dividing table, it is necessary to remove the athlete from the project list, so $b$ is changing, namely the total number cut 1 until $b$ is 0 .

Finally, using comparing fill-bit algorithm to add the contestants on the raceway, end the lane dividing. Comparing fill-bit algorithm is in the process of adding to the track, according to the different units compared its athletes with the pass number of front group or several groups, the same situation is re-selected, otherwise the total number cut one. Highest passes fill method is after filling $\mathrm{n}$ channel, fill the remaining players, the first person removed from the remaining $s$, determines a first set of $c$ channel (namely the $n+1$ channels) whether has person and compared with the first set $1 \sim n$ channel unit is same or not, the same is not filled into this group; and so on, until this group $s$ pass nobody (is empty) and units are not the same, is filled into this group $s$ pass, until the remaining number is zero.

\section{GRouping LANE Dividing SyStem Design}

Grouping lane dividing is a work after the race registration, before the game in the athletic meeting, used to complete the grouping and organization of passes for athletes. Design of grouping lane dividing system allows referees to provide the parameters specific settings for each project, the operation is simple, flexible and fast, after setting up, the system automatically grouping arrangement according to competition rules and related data for each of the entries and display arrangement results [6]. Categories of athletic meeting project include field events, long distance track (more than $800 \mathrm{M}$ or track events), a short distance track (800M following track events, including $800 \mathrm{M}$ ). In the athletic meeting management system, "short distance track" projects require grouping lane dividing (each group has 8 passes), "long-distance track" projects only grouping, "Tin Cup" project also only grouping (based on site grouping in field events). Its system flow chart is shown in Fig. 1.

In the system, the implementation of grouping lane dividing mainly by the number, the total pass rounding, taking the random number, comparing and search algorithms in data structures, and then using the processing result to index or sort in accordance with the number of belonging group, make the same group athletes together and use the random number passes assignment to complete auto passes [7,8]. Grouping lane dividing system mainly completes the grouping lane dividing in short distance track events and the grouping of remote track and field events. Firstly enter the system, then prompted wether grouping lane dividing, if the answer is "Yes", to clear all grouping lane dividing table, and then grouping lane dividing. Click the "short distance track" to grouping lane dividing for the project category that is $800 \mathrm{M}$ and $800 \mathrm{M}$ following items. Click the "distance track and field events" to grouping lane dividing for the project category that is over $800 \mathrm{M}$ and track and field events. Click "Merge" make all grouping lane dividing results into a table in order to print, browse and search. 


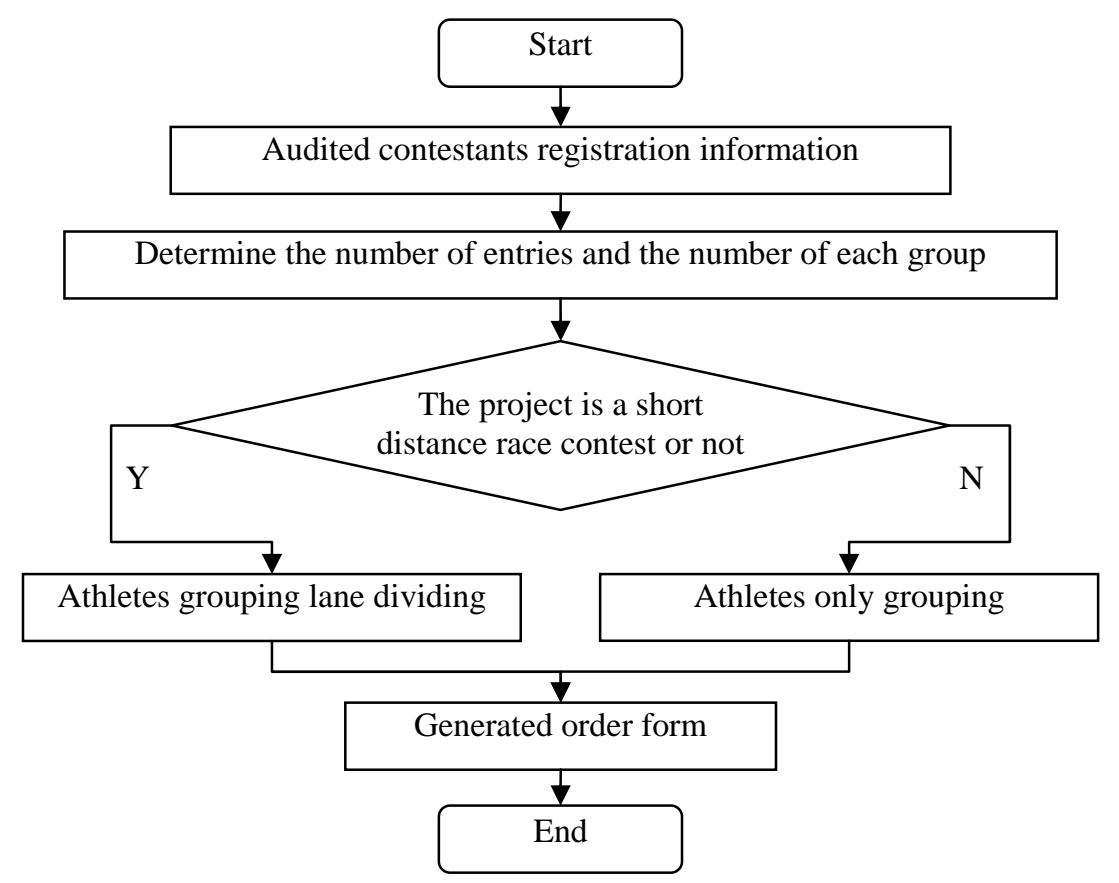

Figure 1: System flow chart

\section{DATABASE DESIGN}

Traditionally, a good database does not mean that there is a good application system, if cannot design a rational database model will not only increase the programming and maintenance difficulty on the side of client and server, but also affects the actual running performance of the system. Its design principles include the following aspects [9], (1) naming standardization, mainly for unified information, ensure the consistency and effectiveness of the system data; (2) the integrity principle, mainly refers to the accuracy and compatibility of the data; (3) the security principle, mainly refers to protect data from unauthorized using, altering and revealing; (4) the principle of scalability, mainly to fully consider the needs of development and transplants, has good expansibility, scalability and moderate redundancy; (5) the principles of standardization, mainly refers to following normalization theory in the database design process, if the degree of standardization is low, there may be a insertion, deletion anomalies, complex modify, data redundancy, and other problems, the solution is to normalize relations model, converted into high-level paradigms.

Database design of this study is mainly the logical design of grouping lane dividing system, mainly in the form of a table to reflect. In which track grouping lane dividing table is used to grouping lane dividing for early and late in the game the game within 800 meters track events, according to the project information and other information in the athlete table removed the corresponding qualifying athletes grouping lane dividing and stored it in the table. Some table structures are shown in Table 1.

TABLE 1: TABLES STRUCTURE OF IN THE DATABASE

\begin{tabular}{l|clcc}
\hline Table name & Fields no & Fields name & Fields type & Fields width \\
\hline & 1 & ID & varchar & 30 \\
& 2 & AthleteName & varchar & 20 \\
Track grouping & 3 & Sex & varchar & 2 \\
lane dividing & 4 & ZuBie & varchar & 10 \\
& 5 & DaoCi & varchar & 10 \\
& 6 & ZuName & varchar & 100 \\
\hline & 1 & ID & varchar & 30 \\
Track and field & 2 & AthleteName & varchar & 20 \\
events grouping & 3 & Sex & varchar & 2 \\
& 4 & CSZuBie & varchar & 10 \\
& 5 & ZuName & varchar & 100 \\
& 6 & $\cdots \cdots$ & $\cdots$ & $\cdots$ \\
\hline
\end{tabular}




\section{V.CONCLUSIONS}

Looking at the big athletic meeting at home and abroad such as the International Olympic Games, Asian Games, the country's National Games, etc., they are integrated into the computer technology, network technology, database technology to manage information, the management of athletic meeting not only includes athlete management, enrolment management, competition score entry, data maintenance, information collection and also includes grouping lane dividing management in competition project $[10,11]$, in the traditional grouping lane dividing management, not only has tedious work, but also consumes more time, so the grouping lane dividing algorithm proposed in this paper and its system design not only increases the flexibility and adaptability of athletic meeting systems, but also greatly reduces the labor intensity of the staff and the athletic meeting organizers, at the same tine make the athletic meeting organization and management become simple, efficient, and flexible [8], and has a significance for the running of the athletic meeting.

\section{ACKNOWLEDGEMENTS}

This work is supported by social science fund project of Liaoning province (L13BTY004), Social Science Planning Foundation project of Liaoning province (No. L13BXW006).

\section{REFERENCES}

[1] H. J. Hu. Track and Field Sports Scheduling Management System Design and Implementation. Master's Degree of University of Electronic Science and Technology, 2007.

[2] J. L. Xi, D. Meng. On the Web-based Management System of General Sports Meet. Journal of Capital Institute of Physical Education, 22(1), pp. 91-93, 2010.

[3] Baidu Encyclopedia, Sports meeting. http://baike.baidu.com/link?url=n6PXv_H0QG2s_79PZm0vlksukV JEcug8VWfyDPa2wjyywwMTB6LZfdb_MFCv_Wn4XeRXNXJCcV 7P822e0clG-_, 2015-1-11.

[4] F. R. Gao, J. L. Yin, J. Zhang. Design and Realization of a Dynamic Grouping Algorithm Dynamic Grouping Algorithm Based on Track Events. Computer Engineering \& Software, 31(1), pp. 1-3, 2011.

[5] B. P. Wei. The Management System of Atheletic Sports. Master's Degree of Xi'an University of Technology, 2006.

[6] J. X. Yang. College Track and Field Games Management Systems Development. Microcomputer Applications, 17(10), pp. 21-23, 2001.

[7] D. K. Liu. Design and Implementation of Integrated Information Management System of Sports Meeting in High School. Master's Degree of Chongqing University, 2009.

[8] J. K. Zhang. The Computer Management System of Small and Medium-sized Track Meeting. Journal of Hebei Normal University(Natural Science Edition), 36(2), pp. 213-216, 2012.

[9] X. L. Zhang, R. Z. Huang, J. Long. Database Theory and Design. People Post Press, 2002.

[10] B. P. Wei, Universities Games Information Management System. Master's Degree of Southwest Petroleum University, 2012.

[11] M. H. Cao, F. Y. Li, J. P. Chu. Design and Implementation of Sports Meeting Management Information System. Journal of Shandong Jiaotong University, 15(4), pp. 77-81, 2007. 